\title{
Blood pressure and hypertension prevalence among oldest-old in China for 16 year: based on CLHLS
}

\author{
Jiajun $\mathrm{Du}^{1 \dagger}$, Guoning Zhu ${ }^{2 \dagger}$, Yanhong Yue ${ }^{3 \dagger}$, Miao Liu ${ }^{4^{*}}$ (D) and Yao He
}

\begin{abstract}
Background: There were little national data on hypertension based on the oldest-old, and lack of information on chronological changes. This study aimed to describe trends of blood pressure (BP) levels and hypertension prevalence for the past 16 years among the oldest-old in China.

Methods: All the oldest-old who had participated in the Chinese Longitudinal Healthy Longevity Survey (CLHLS) 1998 to 2014 with information about BP levels and hypertension were included in the analysis.

Results: There was fluctuation over the past 16 years for both SBP and DBP levels. The mean SBP level decreased from $148.4 \pm 24.4 \mathrm{mmHg}$ in 1998 to $130.8 \pm 18.7 \mathrm{mmHg}$ in 2005, and then increased to $139.7 \pm 22.0 \mathrm{mmHg}$ in 2014 . The mean DBP level decreased from $84.3 \pm 13.4 \mathrm{mmHg}$ in 1998 to $78.9 \pm 11.7 \mathrm{mmHg}$ in 2008 , and then increased to $79.7 \pm 11.8 \mathrm{mmHg}$ in 2014 . The hypertension prevalence increased from 43.1 to $56.5 \%$ for the 16 years. The prevalence of isolated systolic hypertension was lowest in 2002-2005 (14.3\%), and then increased to 30.7\% in 2014. Multivariate logistic regression showed that older age, lower education and economic level, without health insurance were associated with higher hypertension prevalence.
\end{abstract}

Conclusions: There was a significant increase in hypertension prevalence among the Chinese oldest-old from 1998 to 2014. Greater efforts are needed for hypertension prevention among this specific population.

Keywords: Blood pressure, Hypertension, Prevalence, Oldest-old, Epidemiology

\section{Background}

Hypertension is one of the important risk factors for cardiovascular disease. The higher the blood pressure (BP), the greater the coronary heart disease and stroke risks [1-3]. Therefore, it is particularly important to understand the epidemic trend of hypertension. The prevalence of hypertension varies greatly among different age groups, especially among elderly. And isolated systolic hypertension (ISH) (systolic blood pressure (SBP) $\geq 140$ $\mathrm{mmHg}$ while diastolic blood pressure (DBP) $<90 \mathrm{mmHg}$ ) was most existed in elderly $[4,5]$.

\footnotetext{
* Correspondence: liumiaolmbxb@163.com

†iajun Du, Guoning Zhu and Yanhong Yue contributed equally to this work. ${ }^{4}$ Beijing Key Laboratory of Aging and Geriatrics, National Clinical Research Center for Geriatrics Diseases, Institute of Geriatrics, State Key Laboratory of Kidney Diseases, Second Medical Center of Chinese PLA General Hospital, Beijing, China

Full list of author information is available at the end of the article
}

On the other hand, there was a lack of basic data about hypertension prevalence among the oldest-old (aged 80 and over) in China. Previous studies were either among adults, or with small sample, or mainly with inpatients [6, 7]. There were little national data on hypertension based on the oldest-old. Additionally, most studies were based on one time survey, the chronological changes had never been reported, which was a reflection of effects about national control measures of hypertension.

Therefore, we reported the epidemiology characteristics of hypertension based on 63 thousand oldest-old from seven waves (1998, 2002, 2002, 2005, 2008, 2011, 2014) of Chinese Longitudinal Healthy Longevity Survey (CLHLS), the first and largest longitudinal survey focused on the oldest-old in China [8]. We evaluated the prevalence of hypertension by geography and subpopulations, and the chronological changes.

(C) The Author(s). 2019 Open Access This article is distributed under the terms of the Creative Commons Attribution 4.0 International License (http://creativecommons.org/licenses/by/4.0/), which permits unrestricted use, distribution, and reproduction in any medium, provided you give appropriate credit to the original author(s) and the source, provide a link to the Creative Commons license, and indicate if changes were made. The Creative Commons Public Domain Dedication waiver (http://creativecommons.org/publicdomain/zero/1.0/) applies to the data made available in this article, unless otherwise stated. 


\section{Methods}

\section{Study design}

All the participants were from the seven waves of CLHLS, and those who aged more than 80 years old with complete records on BP and hypertension information were included. General characteristic of the seven survey waves was listed in Table 3 in Appendix. The details of the CLHLS and sample design have been described elsewhere [8]. The follow-up survey waves were conducted in 2000, 2005, 2008, 2011, and 2014. The use of CLHLS data was approved by the Biomedical Ethics Committee of Peking University, and written informed consent was obtained from each respondent.

\section{Definitions}

According to the BP levels, participants were divided into the following groups: normal $\mathrm{BP}, \mathrm{SBP} \leq 120 \mathrm{mmHg}$ and DBP $\leq 80 \mathrm{mmHg}$ among those who had never been diagnosed with hypertension; high-normal BP, 120 $\mathrm{mmHg}<\mathrm{SBP} \leq 139 \mathrm{mmHg}$ or $80 \mathrm{mmHg}<\mathrm{DBP} \leq 89 \mathrm{mmHg}$ among those who had never been diagnosed with hypertension; Hypertension, $\mathrm{SBP} \geq 140 \mathrm{mmHg}$ or $\mathrm{DBP} \geq 90$ $\mathrm{mmHg}$ or self-reported being diagnosed as hypertension by II\&III grade hospital before; ISH was defined as SBP $\geq$ $140 \mathrm{mmHg}$ and $\mathrm{DBP}<90 \mathrm{mmHg}$ regardless of previous hypertension diagnosis history. Mean arterial pressure (MAP) was calculated as the following formula: (SBP+
$(2 \times \mathrm{DBP})) \div 3$. Pulse pressure $(\mathrm{PP})$ was calculated as SBP minus DBP.

\section{Statistical analysis}

Mean SBP, DBP, MAP, and PP levels were calculated and expressed as mean \pm standard deviation (SD). Variance analysis was used to compare the differences among subgroups. Besides, we estimated the prevalence of hypertension among all the participants. The age and gender adjusted prevalence of hypertension for the first four waves (1998, 2000, 2002, 2005) was calculated using the direct methods based on the fifth Chinese national census data, and the age and gender adjusted prevalence of hypertension for the last three waves (2008, 2011, 2014) was calculated using the direct methods based on the fifth Chinese national census data. Multivariate logistic regression was used to calculate Odds ratios (ORs) and their $95 \%$ confidence intervals (CIs).

\section{Ethical consideration}

The use of CLHLS data was approved by the Biomedical Ethics Committee of Peking University.

\section{Results}

Trends of BP levels

Figure 1 showed the trends of BP levels among the seven waves. The mean SBP level decreased from 148.4 \pm 24.4
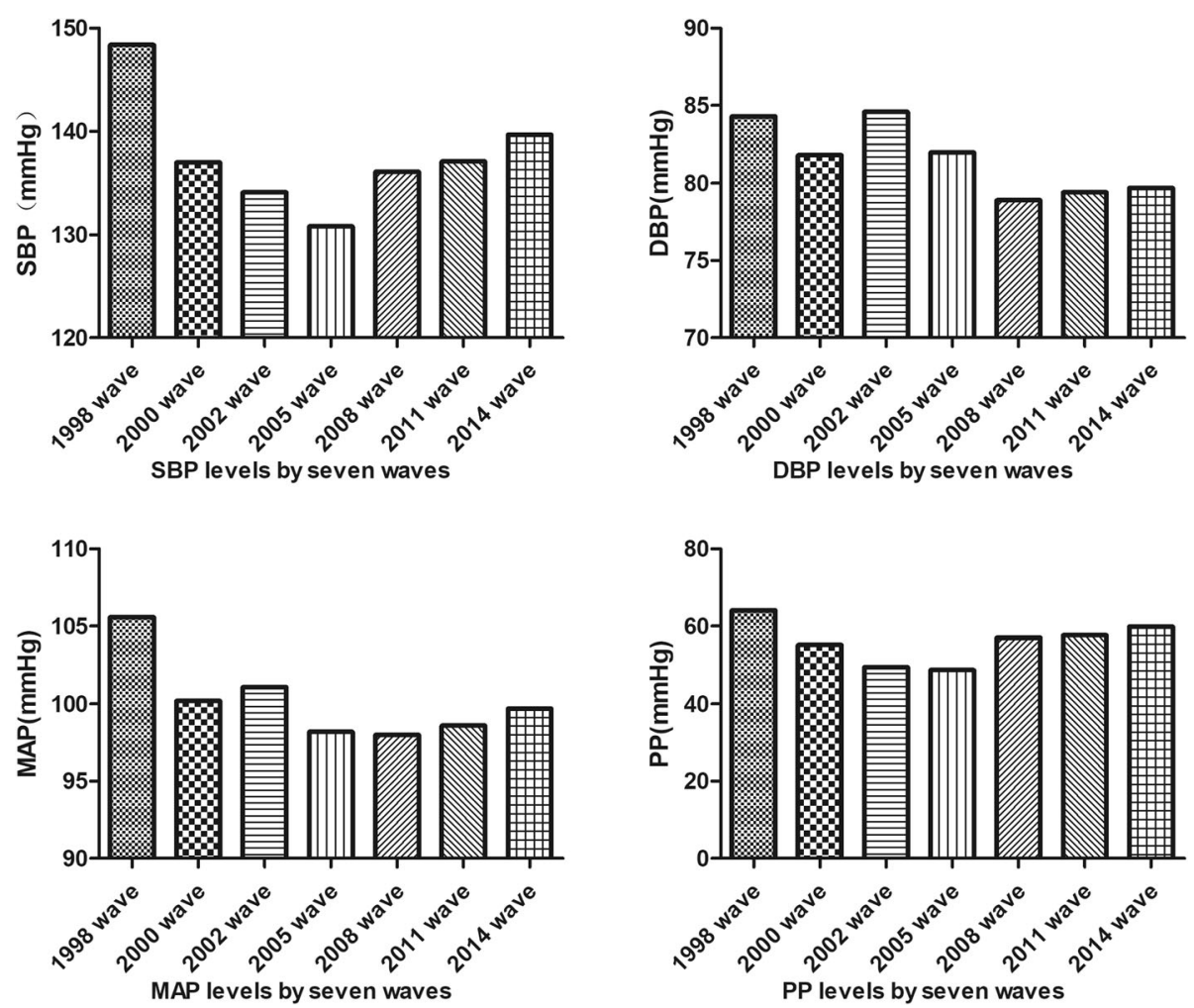

Fig. 1 SBP, DBP MAP and PP levels for the seven survey waves 
$\mathrm{mmHg}$ in 1998 to $130.8 \pm 18.7 \mathrm{mmHg}$ in 2005 , and then increased to $139.7 \pm 22.0 \mathrm{mmHg}$ in 2014 . There was fluctuation over the past 16 years for SBP levels. For different age groups, those aged $80-89$ years had the highest SBP levels, while those aged $\geq 100$ years had the lowest SBP levels $(\mathrm{p}<0.05)$. Compared with different age groups, SBP level was the highest in 80-year-olds and lowest in 100-year-olds ( $\mathrm{p}$ for trend $<0.05$ ). For different categories of residence, those from rural areas had the highest SBP levels, while those from the city had the lowest SBP levels. This trend was pronounced after 2008 wave $(\mathrm{p}<0.05)$. There was no significant difference for male and female $(p>0.05)$. When we excluded those who had hypertension, the trend was similar to that of total population (Table 4 in Appendix). The mean DBP level decreased from $84.3 \pm 13.4$ $\mathrm{mmHg}$ in 1998 to $78.9 \pm 11.7 \mathrm{mmHg}$ in 2008 , and then increased to $79.7 \pm 11.8 \mathrm{mmHg}$ in 2014. Data showed that there had been fluctuations in the seven survey waves during the 16 years (Table 5 in Appendix). The mean MAP level was $105.6 \pm 15.2 \mathrm{mmHg}$ in 1998 wave. And it showed decreasing trend until 2008 survey wave (the lowest mean MAP level was $98.0 \pm 12.3 \mathrm{mmHg}$ ). Then it went up to $99.7 \pm 13.3$ $\mathrm{mmHg}$ in 2014 wave (Table 6 in Appendix). The mean PP level was $64.1 \pm 19.8 \mathrm{mmHg}$ in 1998 wave. And it showed decreasing trend until 2005 survey wave. Then it went up to $59.9 \pm 18.8 \mathrm{mmHg}$ in 2014 wave (Table 7 in Appendix). There was no significant gender difference for DBP, MAP or PP levels, just like SBP levels. The differences among age, category of residence were similar with SBP levels.

\section{Trends in hypertension prevalence}

In 1998 wave, $43.1 \%$ (95\%CI: 42.0-44.1\%) of the participants had hypertension (Table 1). And the prevalence showed an increasing trend along with seven survey waves over the past 16 years $(\mathrm{p}<0.001)$. The weighted prevalence (two weighted method: 1 . weight calculated based on age-sex-residence-specific distribution from the CLHLS study; 2 . weight was calculated based on the fifth (for the first four waves, 1998, 2002, 2002, 2005) and sixth (for the last three waves, 2008, 2011, 2014) national census data.) was similar like unadjusted initial value, with the same trend. Similar to BP levels, there was also a significant decrease trend along with age groups $(\mathrm{p}<0.05)$, relatively higher prevalence in rural areas and eastern China $(p<0.05)$, and no significant difference for male and female $(p>0.05)$.

The trend for ISH prevalence was different. The lowest prevalence was in 2002 wave (14.3, 95\%CI: $13.7-15.0 \%$ ), the highest prevalence was $30.5 \%$ (95\% CI: $29.6-31.5 \%)$ in 1998 wave and 30.7\% (95\%CI: $29.3-32.0 \%$ ) in 2014 wave. The differences among gender, age, category of residence were similar to that of BP levels (Table 8 in Appendix).

The prevalence of high-normal BP also showed an increasing trend. It went from $20.9 \%$ (95\%: $20.0-21.7 \%)$ in

Table 1 The prevalence (\%) of hypertension by seven waves

\begin{tabular}{|c|c|c|c|c|c|c|c|c|}
\hline Wave & 1998 & 2000 & 2002 & 2005 & 2008 & 2011 & 2014 & $p$ \\
\hline \multicolumn{9}{|l|}{ Gender } \\
\hline Male & $43.1(41.4-44.7)$ & $43.3(41.9-44.8)$ & $42.3(40.9-43.9)$ & $48.8(47.3-50.3)$ & $46.3(44.8-47.8)$ & $50.1(48.1-52.1)$ & $55.3(53.0-57.5)$ & $<0.001$ \\
\hline Female & $43.1(41.7-44.4)$ & $43.8(42.6-45.1)$ & $43.4(42.3-44.6)$ & $46.4(45.2-47.6)$ & $45.8(44.6-47.0)$ & $54.6(53.0-56.2)$ & $57.4(55.5-59.3)$ & $<0.001$ \\
\hline$p$ & 0.995 & 0.607 & 0.277 & 0.016 & 0.602 & $<0.001$ & 0.150 & \\
\hline \multicolumn{9}{|l|}{ Age-group } \\
\hline $80-89$ yrs & 45.4(43.7-47.1) & $44.3(42.9-45.7)$ & $44.5(43.0-46.0)$ & $51.3(49.8-52.9)$ & $50.2(48.6-57.1)$ & $56.7(54.8-58.6)$ & 59.9(57.9-62.0) & $<0.001$ \\
\hline 90-99yrs & $43.9(42.1-45.7)$ & $44.3(42.7-45.9)$ & $43.5(42.7-45.9)$ & $46.9(45.4-48.5)$ & $46.4(44.9-47.9)$ & $52.6(50.6-46.6)$ & $54.3(51.8-56.7)$ & $<0.001$ \\
\hline $100-y r s$ & $38.7(36.7-40.6)$ & $41.2(39.2-43.2)$ & $40.5(38.8-42.3)$ & $42.3(40.4-44.1)$ & $39.9(38.8-42.3)$ & $46.0(43.4-48.6)$ & 52.2(48.9-55.6) & $<0.001$ \\
\hline$p_{\text {for trend }}$ & $<0.001$ & 0.032 & 0.003 & $<0.001$ & $<0.001$ & $<0.001$ & $<0.001$ & \\
\hline \multicolumn{9}{|c|}{ Category of residence } \\
\hline City & $41.8(40.1-43.5)$ & $43.2(41.7-45.1)$ & $43.0(41.1-44.9)$ & $45.9(44.6-47.2)$ & $43.7(41.7-45.8)$ & $48.8(43.8-51.7)$ & $51.1(41.2-55.1)$ & $<0.001$ \\
\hline Town & & $43.4(41.5-44.9)$ & $43.0(41.3-45.1)$ & $48.4(46.5-50.3)$ & $46.1(44.9-47.3)$ & $51.0(48.8-53.3)$ & $56.2(53.7-58.8)$ & $<0.001$ \\
\hline Rural & $43.8(42.3-45.3)$ & $44.2(41.7-46.3)$ & $43.2(41.7-44.3)$ & $50.0(47.8-52.0)$ & $48.9(46.9-51.0)$ & $55.1(53.4-56.8)$ & $58.0(56.1-59.9)$ & $<0.001$ \\
\hline$p_{\text {for trend }}$ & 0.123 & $<0.001$ & 0.634 & $<0.001$ & $<0.001$ & 0.006 & 0.838 & \\
\hline Total & $43.1(42.0-44.1)$ & $43.6(42.7-44.5)$ & $43.0(42.1-43.9)$ & $47.3(46.4-48.3)$ & $46.0(45.1-46.9)$ & $52.8(51.6-54.0)$ & $56.5(55.1-58.0)$ & $<0.001$ \\
\hline Weighted Total $^{\dagger}$ & $45.2(44.2-46.3)$ & $44.8(43.9-45.8)$ & $44.4(42.4-46.4)$ & $50.6(48.6-52.6)$ & $50.2(48.2-52.2)$ & $53.8(51.6-55.9)$ & $57.4(55.1-59.7)$ & $<0.001$ \\
\hline Weighted Total $^{\dagger}$ & $45.3(43.8-46.9)$ & $44.3(43.1-45.6)$ & $44.6(43.2-45.9)$ & $50.9(49.5-52.3)$ & $49.8(48.5-51.3)$ & $56.6(54.8-58.3)$ & $59.5(57.6-61.4)$ & $<0.001$ \\
\hline
\end{tabular}

*:City and town were combined as one category in 1998 wave

${ }^{\dagger}$ : Weight was calculated based on age-sex-residence-specific distribution from the CLHLS study

${ }^{\ddagger}$ : Weight was calculated based on the sixth national census data 
1998 wave to the highest of $47.5 \%$ (95\%: 46.6-48.4\%) in 2008 wave. Then it remained at about $35.0 \%$ in the following two waves. For different stages of hypertension, participants with hypertension who were classified as stage I or stage II also showed similar increasing trend (Fig. 2).

Sensitivity analysis was performed among those who were first included in the analysis. The BP levels, the prevalence of hypertension were a little higher than the results of the total participants in each survey wave (Table 9 in Appendix).

\section{Multivariable analysis of hypertension prevalence}

Using the most current survey wave data (CLHLS 2014), Table 2 presented the adjusted ORs and 95\%CI for the association between covariates in four multivariable logistic regressions each using the following two binary outcomes as dependent variables: (1) prevalence of hypertension; (2) prevalence of ISH. Gender, marriage status had no statistical association with hypertension prevalence. Compared with Han nationality, minorities had lower hypertension prevalence $(\mathrm{OR}=0.76,95 \% \mathrm{CI}$ : 0.59-0.99). However, only
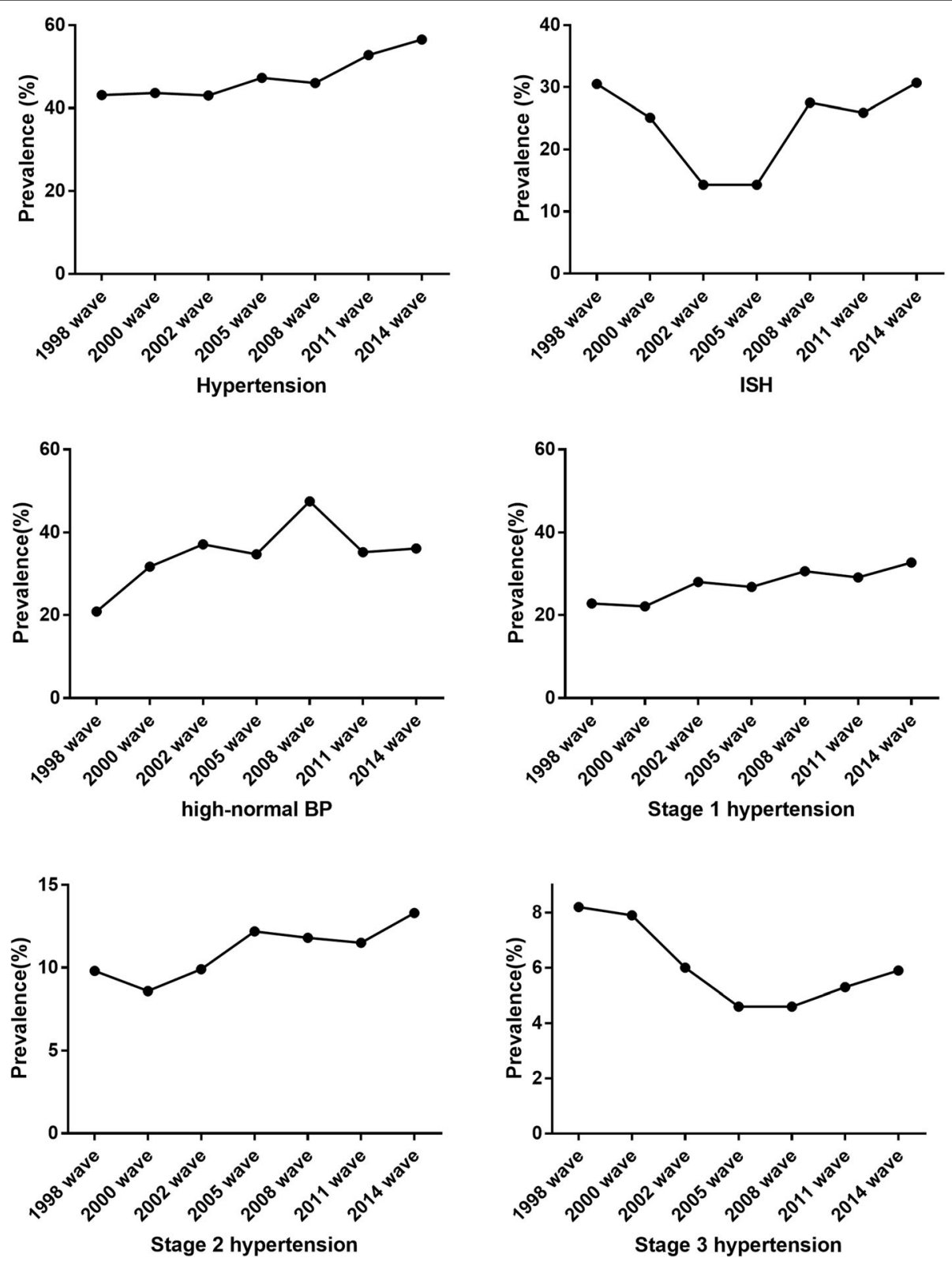

Fig. 2 Prevalence of hypertension for the seven survey waves 
Table 2 Adjusted ORs (95\%Cl) for prevalence, of hypertension

\begin{tabular}{|c|c|c|}
\hline Characteristic & Prevalence & $\begin{array}{l}\text { Prevalence of isolated } \\
\text { systolic hypertension }\end{array}$ \\
\hline \multicolumn{3}{|l|}{ Gender } \\
\hline Male & 1.00 (ref) & 1.00 (ref) \\
\hline Female & $1.03(0.82-1.29)$ & $0.96(0.80-1.14)$ \\
\hline \multicolumn{3}{|l|}{ Age-group } \\
\hline $80-89$ yrs & 1.00 (ref) & 1.00 (ref) \\
\hline 90-99yrs & $0.69(0.58-0.79) *$ & $0.99(0.86-1.15)$ \\
\hline $100-y r s$ & $0.52(0.42-0.64) *$ & $0.98(0.82-1.18)$ \\
\hline \multicolumn{3}{|l|}{ Education years } \\
\hline 0 years & 1.00 (ref) & 1.00 (ref) \\
\hline $1-6$ years & $1.06(0.89-1.27)$ & $1.03(0.87-1.22)$ \\
\hline$\geq 7$ years & $1.19(0.79-1.63)$ & $0.87(0.65-1.17)$ \\
\hline \multicolumn{3}{|l|}{ Current marriage } \\
\hline Married & 1.00 (ref) & 1.00 (ref) \\
\hline Divorced/Widowhood/other & $1.10(0.93-1.30)$ & $1.05(0.89-1.23)$ \\
\hline \multicolumn{3}{|l|}{ Nationality } \\
\hline Han & 1.00 (ref) & 1.00 (ref) \\
\hline Minority & $0.76(0.56-0.99) *$ & $0.86(0.66-1.12)$ \\
\hline \multicolumn{3}{|l|}{ Smoking } \\
\hline Never smoking & 1.00 (ref) & 1.00 (ref) \\
\hline Ever smoking & $1.36(1.08-1.70)^{*}$ & $0.93(0.75-1.16)$ \\
\hline Current smoking & $1.03(0.82-1.30)$ & $0.92(0.74-1.14)$ \\
\hline \multicolumn{3}{|l|}{ Alcohol drinking } \\
\hline Never drinking & 1.00 (ref) & 1.00 (ref) \\
\hline Ever drinking & $1.21(0.96-1.54)$ & $0.89(0.70-1.13)$ \\
\hline Current drinking & $0.81(0.66-1.02)$ & $0.88(0.71-1.09)$ \\
\hline \multicolumn{3}{|l|}{ Central obesity } \\
\hline No & 1.00(ref) & 1.00 (ref) \\
\hline Yes & $1.76(1.52-2.03)^{*}$ & $1.46(1.27-1.68)^{*}$ \\
\hline \multicolumn{3}{|l|}{ Category of residence } \\
\hline City & 1.00 (ref) & 1.00 (ref) \\
\hline Town & $1.13(1.09-1.34)^{*}$ & $1.21(0.98-1.51)$ \\
\hline Rural & $1.24(1.08-1.47)^{*}$ & $1.24(1.01-1.53)^{*}$ \\
\hline \multicolumn{3}{|l|}{ Having health insurance } \\
\hline No & 1.00 (ref) & 1.00 (ref) \\
\hline Yes & $1.05(0.88-1.24)$ & $0.86(0.74-1.01)$ \\
\hline
\end{tabular}

${ }^{*}: p<0.05$

participants aged 90-99 and $\geq 100$ years old were of lower risk of hypertension than those aged 80-89 years old $(\mathrm{OR}=0.69, \quad 95 \% \mathrm{CI}: 0.58-0.79 ; \quad \mathrm{OR}=0.52$, 95\%CI: 0.42-0.64 respectively). Participant who had higher education levels, central obesity, were more likely to have hypertension. For different categories of residence, compared with those from urban (including city and town) areas, those from rural areas had higher risk of prevalence of hypertension $(\mathrm{OR}=1.24$, 95\%CI: $1.08-0.99 ; \quad$ OR $=0.53, \quad 95 \% \mathrm{CI}: \quad 0.35-0.82$ respectively).

\section{Discussion}

Our study provided the mean BP levels and epidemic picture of hypertension among the oldest-old in China based on CLHLS 1998 to 2014 survey wave. The results 
indicated that BP levels were high especially DBP and PP levels. Besides, hypertension was of relatively high prevalence, and showed an increasing trend along with seven survey waves over the past 16 years.

The study of BP levels had filled in the blanks for mean BP levels based on community oldest-old in China. And there was fluctuation over the past 16 years for both SBP and DBP levels.. The mean BP levels were significantly higher than those of adults while they were about the same as those of the younger elderly who aged 65-74 years old from the interASIA study in 2000-2001 $[9,10]$. The above results and the comparison with the domestic and foreign studies indicated that the BP levels of the oldest-old in China was close to those of the younger elderly, which were lower than those of the elderly in the same age group in developing countries. However what cannot be ignored was that the increasing trend of BP levels for the past 16 years, which was worth paying attention to.

The prevalence of hypertension has gradually increased for the past 16 years was consistent with hypertension prevalence and increasing cardiovascular disease burden. And compared with previous studies based adults or younger elderly, the oldest-old had the highest prevalence [11]. Our study also provides data on ISH, which was an important subtype of serious harm among elderly. Data showed that about half of the hypertension oldest-old were classified as ISH subtype, which was higher than the situation in other age groups [12-14]. The result from our study showed that there was no less than $30 \%$ percent of total hypertension patients could be classified as stage II and above. This suggested that we need to pay attention to the serious situation of high ISH prevalence and high percentage of stage II \& III hypertension in the oldest-old and prevent subsequent cardiovascular diseases.

In addition, it is worth noting that not only the hypertension prevalence was increasing, the prevalence of high-normal BP also showed an increasing trend. And evidence showed that high-normal BP was one of the important risk factors of hypertension and cardiovascular diseases [15]. Without enough early prevention measures, those who had high-normal BP will sooner or later develop into hypertension.

We also added information about related factors associated with hypertension (including ISH) prevalence. The gender difference was not significant, which was unlike adults and in line with expectations, since the protective effect of estrogen had disappeared for more than 30 years[16-19]. For unhealthy lifestyles, those who were ever smoking or alcohol drinking had higher prevalence. This was in line with the phenomenon of quitting smoking or stop drinking because of disease [20]. Obesity was a risk factor for hypertension, just like other studies about adults. Evidence showed that there were a series of endocrine and metabolic changes due to obesity, which might be associated with the hypertension [21].

There were several strengths. First, the CLHLS study was a large scale nationwide study covering 23 provinces of China for the past 16 years. The large sample was unique for representation of the oldest-old. Second, there was good design and strict quality control during the whole survey, which ensures the good quality data.

Our study had several limitations. First, the study sample was from 23 provinces, there was a lack of representativeness for other unselected provinces. Besides, because of the sampling method used in CLHLS, the participants were not representative samples. We calculated both the crude and weighted prevalence, and the trends were similar. Second, most of the participants of CLHLS were from communities, and there was only less than $5 \%$ who lived in living in nursing homes or other institutions. But this was in accordance with the situation in China, since more than $95 \%$ of the elderly were home-based care. Third, there was less than 3.5\% frail oldest-old with severe diseases or disability who didn't have BP recorded, and this may cause underestimation of prevalence. Fourth, BP levels were measured on the same day. Although the average of two times was use, long-time changes were not captured. Fifth, we didn't have information about treatment and medication information. Although this kind of definition had been adopted in quite a number of epidemiological investigations, the resulting bias cannot be ignored. Combined with the results of previous studies on treatment rates in China and the prevalence of hypertension in this study, this bias may lead to an overestimation. However, taking 2014 survey as an example, there were a total of 299 participants who were classified as hypertension according to previous history with $\mathrm{SBP} \leq 140 \mathrm{mmHg}$ and $\mathrm{DBP} \leq 90$ $\mathrm{mmHg}$ this time. And the misclassification participants were calculated as $299-299 * 30 \%$ (the treatment rate according to the China PEACE Million Persons Project) $=$ 209 , and the misclassification rate $=209 / 4587=4.6 \%$. this number times. Sixth, only cross-sectional analysis was conducted, and there was lack of the lack of survival data analysis and sensitivity analysis in relation to survival of at least 1-2 years. Seventh, due to the cross-sectional nature, the results about risk factor of prevalence were of low evidence level.

\section{Conclusions}

In conclusion, this was the first large scale nationwide study about hypertension with long time comparisons for over 16 years among oldest-old in Chinese. The results also provided evidence about the trends of hypertension prevalence in China, which indicated that hypertension prevention was still a long and arduous task. 


\section{Appendix}

Table 3 General characteristic of the seven survey waves

\begin{tabular}{lllllll}
\hline Wave & Total population & Total oldest-old & Excluded oldest-old due to missing information & Included population & Mean age & Male (\%) \\
\hline 1998 & 9093 & 8959 & 265 & 8694 & $92.3 \pm 7.6$ & 39.7 \\
2000 & 11,200 & 11,162 & 354 & 10,808 & $91.3 \pm 7.5$ & 41.6 \\
2002 & 16,064 & 11,175 & 102 & 11,073 & $92.6 \pm 7.6$ & 39.4 \\
2005 & 15,638 & 16,658 & 700 & 10,498 & $92.8 \pm 7.2$ & 39.2 \\
2008 & 16,540 & 12,002 & 101 & 11,301 & $92.7 \pm 7.4$ & 39.4 \\
2011 & 9765 & 6530 & 129 & 6401 & $92.2 \pm 7.7$ & 40.2 \\
2014 & 7192 & 4738 & 151 & 4587 & $91.3 \pm 7.6$ & 41.3 \\
\hline
\end{tabular}


Table 4 SBP levels by seven waves

\begin{tabular}{|c|c|c|c|c|c|c|c|c|c|}
\hline Variable & Wave & 1998 & 2000 & 2002 & 2005 & 2008 & 2011 & 2014 & $p$ \\
\hline \multirow[t]{20}{*}{ SBP } & \multicolumn{9}{|l|}{ Gender } \\
\hline & Male & $\begin{array}{l}147.9 \pm \\
23.6\end{array}$ & $\begin{array}{l}137.5 \pm \\
21.1\end{array}$ & $\begin{array}{l}134.7 \pm \\
17.1\end{array}$ & $\begin{array}{l}131.1 \pm \\
18.1\end{array}$ & $\begin{array}{l}135.5 \pm \\
20.9\end{array}$ & $\begin{array}{l}136.2 \pm \\
20.4\end{array}$ & $\begin{array}{l}139.9 \pm \\
20.8\end{array}$ & $\begin{array}{l}< \\
0.001\end{array}$ \\
\hline & Female & $\begin{array}{l}148.8 \pm \\
25.1\end{array}$ & $\begin{array}{l}137.6 \pm \\
22.3\end{array}$ & $\begin{array}{l}134.3 \pm \\
17.5\end{array}$ & $\begin{array}{l}130.6 \pm \\
19.0\end{array}$ & $\begin{array}{l}136.1 \pm \\
21.3\end{array}$ & $\begin{array}{l}137.1 \pm \\
23.0\end{array}$ & $\begin{array}{l}139.4 \pm \\
22.8\end{array}$ & $\begin{array}{l}< \\
0.001\end{array}$ \\
\hline & $\mathrm{p}$ & 0.078 & 0.825 & 0.195 & 0.064 & 0.181 & 0.102 & 0.100 & \\
\hline & \multicolumn{9}{|c|}{ Age-group } \\
\hline & $\begin{array}{l}80-89 \\
\text { yrs }\end{array}$ & $\begin{array}{l}150.9 \pm \\
24.3\end{array}$ & $\begin{array}{l}138.5 \pm \\
22.3\end{array}$ & $\begin{array}{l}135.5 \pm \\
18.0\end{array}$ & $\begin{array}{l}132.4 \pm \\
18.7\end{array}$ & $\begin{array}{l}138.0 \pm \\
21.8\end{array}$ & $\begin{array}{l}137.8 \pm \\
20.7\end{array}$ & $\begin{array}{l}140.4 \pm \\
20.8\end{array}$ & $\begin{array}{l}< \\
0.001\end{array}$ \\
\hline & $\begin{array}{l}90-99 \\
\text { yrs }\end{array}$ & $\begin{array}{l}148.6 \pm \\
24.0\end{array}$ & $\begin{array}{l}138.0 \pm \\
22.0\end{array}$ & $\begin{array}{l}134.5 \pm \\
17.2\end{array}$ & $\begin{array}{l}131.1 \pm \\
18.7\end{array}$ & $\begin{array}{l}135.5 \pm \\
21.3\end{array}$ & $\begin{array}{l}137.5 \pm \\
22.6\end{array}$ & $\begin{array}{l}139.4 \pm \\
22.4\end{array}$ & $\begin{array}{l}< \\
0.001\end{array}$ \\
\hline & $100-y r s$ & $\begin{array}{l}144.7 \pm \\
25.1\end{array}$ & $\begin{array}{l}135.0 \pm \\
20.3\end{array}$ & $\begin{array}{l}133.1 \pm \\
16.6\end{array}$ & $\begin{array}{l}127.9 \pm \\
18.2\end{array}$ & $\begin{array}{l}133.5 \pm \\
19.8\end{array}$ & $\begin{array}{l}134.5 \pm \\
23.4\end{array}$ & $\begin{array}{l}137.6 \pm \\
23.9\end{array}$ & $\begin{array}{l}< \\
0.001\end{array}$ \\
\hline & $p_{\text {for trend }}$ & $<0.001$ & $<0.001$ & $<0.001$ & $<0.001$ & $<0.001$ & $<0.001$ & 0.007 & \\
\hline & \multicolumn{9}{|c|}{ Category of residence } \\
\hline & City & $\begin{array}{l}148.3 \pm \\
24.0\end{array}$ & $\begin{array}{l}137.2 \pm \\
21.8\end{array}$ & $\begin{array}{l}134.6 \pm \\
18.3\end{array}$ & $\begin{array}{l}130.3 \pm \\
18.7\end{array}$ & $\begin{array}{l}133.8 \pm \\
18.7\end{array}$ & $\begin{array}{l}133.2 \pm \\
20.2\end{array}$ & $\begin{array}{l}134.1 \pm \\
20.0\end{array}$ & $\begin{array}{l}< \\
0.001\end{array}$ \\
\hline & Town & & $\begin{array}{l}137.4 \pm \\
21.3\end{array}$ & $\begin{array}{l}135.1 \pm \\
17.2\end{array}$ & $\begin{array}{l}130.8 \pm \\
18.4\end{array}$ & $\begin{array}{l}134.8 \pm \\
20.5\end{array}$ & $\begin{array}{l}136.7 \pm \\
21.2\end{array}$ & $\begin{array}{l}138.9 \pm \\
20.6\end{array}$ & $\begin{array}{l}< \\
0.001\end{array}$ \\
\hline & Rural & $\begin{array}{l}148.6 \pm \\
24.8\end{array}$ & $\begin{array}{l}138.0 \pm \\
22.1\end{array}$ & $\begin{array}{l}134.2 \pm \\
17.0\end{array}$ & $\begin{array}{l}130.9 \pm \\
18.8\end{array}$ & $\begin{array}{l}136.9 \pm \\
20.1\end{array}$ & $\begin{array}{l}138.4 \pm \\
23.0\end{array}$ & $\begin{array}{l}141.2 \pm \\
22.9\end{array}$ & $\begin{array}{l}< \\
0.001\end{array}$ \\
\hline & $P_{\text {for trend }}$ & 0.587 & 0.277 & 0.087 & 0.496 & $<0.001$ & $<0.001$ & $<0.001$ & \\
\hline & \multicolumn{9}{|c|}{ Region of China } \\
\hline & East & $\begin{array}{l}149.0 \pm \\
24.0\end{array}$ & $\begin{array}{l}139.0 \pm \\
21.1\end{array}$ & $\begin{array}{l}134.6 \pm \\
17.1\end{array}$ & $\begin{array}{l}132.8 \pm \\
21.6\end{array}$ & $\begin{array}{l}139.7 \pm \\
22.7\end{array}$ & $\begin{array}{l}139.2 \pm \\
22.3\end{array}$ & $\begin{array}{l}140.2 \pm \\
22.4\end{array}$ & $\begin{array}{l}< \\
0.001\end{array}$ \\
\hline & Central & $\begin{array}{l}148.1 \pm \\
24.8\end{array}$ & $\begin{array}{l}134.8 \pm \\
22.3\end{array}$ & $\begin{array}{l}134.2 \pm \\
18.4\end{array}$ & $\begin{array}{l}130.6 \pm \\
17.6\end{array}$ & $\begin{array}{l}135.3 \pm \\
20.5\end{array}$ & $\begin{array}{l}134.4 \pm \\
21.8\end{array}$ & $\begin{array}{l}139.8 \pm \\
22.2\end{array}$ & $\begin{array}{l}< \\
0.001\end{array}$ \\
\hline & West & $\begin{array}{l}146.6 \pm \\
25.2\end{array}$ & $\begin{array}{l}133.0 \pm \\
20.9\end{array}$ & $\begin{array}{l}132.2 \pm \\
16.8\end{array}$ & $\begin{array}{l}127.9 \pm \\
16.4\end{array}$ & $\begin{array}{l}135.5 \pm \\
21.9\end{array}$ & $\begin{array}{l}134.0 \pm \\
20.0\end{array}$ & $\begin{array}{l}136.7 \pm \\
19.1\end{array}$ & $\begin{array}{l}< \\
0.001\end{array}$ \\
\hline & $P_{\text {for trend }}$ & 0.006 & $<0.001$ & $<0.001$ & $<0.001$ & $<0.001$ & $<0.001$ & 0.007 & \\
\hline & Total & $\begin{array}{l}148.4 \pm \\
24.4\end{array}$ & $\begin{array}{l}137.0 \pm \\
21.6\end{array}$ & $\begin{array}{l}134.1 \pm \\
17.4\end{array}$ & $\begin{array}{l}130.8 \pm \\
18.7\end{array}$ & $\begin{array}{l}136.1 \pm \\
21.4\end{array}$ & $\begin{array}{l}137.1 \pm \\
22.0\end{array}$ & $\begin{array}{l}139.7 \pm \\
22.0\end{array}$ & $\begin{array}{l}< \\
0.001\end{array}$ \\
\hline \multirow{12}{*}{$\begin{array}{l}\text { SBP among those without } \\
\text { hypertension }\end{array}$} & \multicolumn{9}{|l|}{ Gender } \\
\hline & Male & $\begin{array}{l}135.7 \pm \\
19.5\end{array}$ & $\begin{array}{l}126.3 \pm \\
16.6\end{array}$ & $\begin{array}{l}126.4 \pm \\
14.2\end{array}$ & $\begin{array}{l}120.4 \pm \\
10.7\end{array}$ & $\begin{array}{l}122.7 \pm \\
11.2\end{array}$ & $\begin{array}{l}121.6 \pm \\
11.7\end{array}$ & $\begin{array}{l}123.3 \pm \\
10.9\end{array}$ & $\begin{array}{l}< \\
0.001\end{array}$ \\
\hline & Female & $\begin{array}{l}136.0 \pm \\
21.3\end{array}$ & $\begin{array}{l}125.6 \pm \\
17.4\end{array}$ & $\begin{array}{l}125.2 \pm \\
13.6\end{array}$ & $\begin{array}{l}119.1 \pm \\
11.3\end{array}$ & $\begin{array}{l}122.5 \pm \\
11.1\end{array}$ & $\begin{array}{l}120.7 \pm \\
12.4\end{array}$ & $\begin{array}{l}121.9 \pm \\
11.9\end{array}$ & $\begin{array}{l}< \\
0.001\end{array}$ \\
\hline & $\mathrm{p}$ & $<0.001$ & 0.176 & 0.002 & 0.097 & 0.751 & 0.981 & 0.317 & \\
\hline & \multicolumn{9}{|c|}{ Age-group } \\
\hline & $\begin{array}{l}80-89 \\
\text { yrs }\end{array}$ & $\begin{array}{l}138.5 \pm \\
20.4\end{array}$ & $\begin{array}{l}126.7 \pm \\
17.8\end{array}$ & $\begin{array}{l}126.3 \pm \\
14.2\end{array}$ & $\begin{array}{l}120.4 \pm \\
10.9\end{array}$ & $\begin{array}{l}123.2 \pm \\
11.2\end{array}$ & $\begin{array}{l}122.4 \pm \\
11.5\end{array}$ & $\begin{array}{l}123.9 \pm \\
10.4\end{array}$ & $\begin{array}{l}< \\
0.001\end{array}$ \\
\hline & $\begin{array}{l}90-99 \\
\text { yrs }\end{array}$ & $\begin{array}{l}135.7 \pm \\
20.0\end{array}$ & $\begin{array}{l}126.2 \pm \\
17.2\end{array}$ & $\begin{array}{l}125.8 \pm \\
13.9\end{array}$ & $\begin{array}{l}120.3 \pm \\
10.7\end{array}$ & $\begin{array}{l}122.1 \pm \\
11.3\end{array}$ & $\begin{array}{l}121.2 \pm \\
12.1\end{array}$ & $\begin{array}{l}122.5 \pm \\
11.6\end{array}$ & $\begin{array}{l}< \\
0.001\end{array}$ \\
\hline & $100-y r s$ & $\begin{array}{l}132.8 \pm \\
21.1\end{array}$ & $\begin{array}{l}123.8 \pm \\
15.3\end{array}$ & $\begin{array}{l}124.7 \pm \\
13.4\end{array}$ & $\begin{array}{l}117.7 \pm \\
11.6\end{array}$ & $\begin{array}{l}122.5 \pm \\
10.9\end{array}$ & $\begin{array}{l}119.1 \pm \\
12.8\end{array}$ & $\begin{array}{l}119.6 \pm \\
13.0\end{array}$ & $\begin{array}{l}< \\
0.001\end{array}$ \\
\hline & $P_{\text {for trend }}$ & $<0.001$ & $<0.001$ & 0.001 & $<0.001$ & 0.008 & $<0.001$ & $<0.001$ & \\
\hline & \multicolumn{9}{|c|}{ Category of residence } \\
\hline & City & $\begin{array}{l}135.6 \pm \\
20.3\end{array}$ & $\begin{array}{l}125.8 \pm \\
17.1\end{array}$ & $\begin{array}{l}125.4 \pm \\
13.6\end{array}$ & $\begin{array}{l}118.9 \pm \\
10.8\end{array}$ & $\begin{array}{l}122.3 \pm \\
10.6\end{array}$ & $\begin{array}{l}120.5 \pm \\
12.1\end{array}$ & $\begin{array}{l}120.7 \pm \\
11.3\end{array}$ & $\begin{array}{l}< \\
0.001\end{array}$ \\
\hline & Town & & $\begin{array}{l}125.9 \pm \\
16.4\end{array}$ & $\begin{array}{l}125.6 \pm \\
13.5\end{array}$ & $\begin{array}{l}119.0 \pm \\
11.5\end{array}$ & $\begin{array}{l}122.6 \pm \\
11.0\end{array}$ & $\begin{array}{l}120.9 \pm \\
12.3\end{array}$ & $\begin{array}{l}123.4 \pm \\
11.8\end{array}$ & $\begin{array}{l}< \\
0.001\end{array}$ \\
\hline
\end{tabular}


Table 4 SBP levels by seven waves (Continued)

\begin{tabular}{|c|c|c|c|c|c|c|c|c|c|}
\hline Variable & Wave & 1998 & 2000 & 2002 & 2005 & 2008 & 2011 & 2014 & $p$ \\
\hline & Rural & $\begin{array}{l}136.5 \pm \\
20.8\end{array}$ & $\begin{array}{l}125.9 \pm \\
17.6\end{array}$ & $\begin{array}{l}126.3 \pm \\
15.0\end{array}$ & $\begin{array}{l}120.1 \pm \\
11.1\end{array}$ & $\begin{array}{l}122.7 \pm \\
11.4\end{array}$ & $\begin{array}{l}121.8 \pm \\
11.8\end{array}$ & $\begin{array}{l}122.5 \pm \\
11.4\end{array}$ & $\begin{array}{l}< \\
0.001\end{array}$ \\
\hline & $p_{\text {for trend }}$ & 0.142 & 0.957 & 0.089 & 0.001 & 0.722 & 0.062 & 0.004 & \\
\hline & \multicolumn{9}{|c|}{ Region of China } \\
\hline & East & $\begin{array}{l}136.5 \pm \\
20.1\end{array}$ & $\begin{array}{l}127.4 \pm \\
17.3\end{array}$ & $\begin{array}{l}126.1 \pm \\
13.6\end{array}$ & $\begin{array}{l}122.4 \pm \\
12.1\end{array}$ & $\begin{array}{l}123.5 \pm \\
10.5\end{array}$ & $\begin{array}{l}122.4 \pm \\
11.6\end{array}$ & $\begin{array}{l}123.4 \pm \\
11.4\end{array}$ & $\begin{array}{l}< \\
0.001\end{array}$ \\
\hline & Central & $\begin{array}{l}136.4 \pm \\
21.5\end{array}$ & $\begin{array}{l}123.7 \pm \\
16.9\end{array}$ & $\begin{array}{l}126.0 \pm \\
15.5\end{array}$ & $\begin{array}{l}120.1 \pm \\
10.9\end{array}$ & $\begin{array}{l}122.4 \pm \\
12.1\end{array}$ & $\begin{array}{l}121.9 \pm \\
11.6\end{array}$ & $\begin{array}{l}122.6 \pm \\
11.4\end{array}$ & $\begin{array}{l}< \\
0.001\end{array}$ \\
\hline & West & $\begin{array}{l}133.5 \pm \\
20.6\end{array}$ & $\begin{array}{l}123.7 \pm \\
14.7\end{array}$ & $\begin{array}{l}123.7 \pm \\
12.1\end{array}$ & $\begin{array}{l}118.5 \pm \\
10.5\end{array}$ & $\begin{array}{l}120.7 \pm \\
11.9\end{array}$ & $\begin{array}{l}118.8 \pm \\
12.8\end{array}$ & $\begin{array}{l}121.8 \pm \\
11.5\end{array}$ & $\begin{array}{l}< \\
0.001\end{array}$ \\
\hline & $P$ for trend & 0.001 & $<0.001$ & $<0.001$ & $<0.001$ & $<0.001$ & $<0.001$ & 0.179 & \\
\hline & Total & $\begin{array}{l}136.0 \pm \\
20.6\end{array}$ & $\begin{array}{l}125.7 \pm \\
16.9\end{array}$ & $\begin{array}{l}119.7 \pm \\
11.1\end{array}$ & $\begin{array}{l}119.6 \pm \\
11.1\end{array}$ & $\begin{array}{l}122.6 \pm \\
11.2\end{array}$ & $\begin{array}{l}121.1 \pm \\
12.1\end{array}$ & $\begin{array}{l}122.5 \pm \\
11.5\end{array}$ & $\begin{array}{l}< \\
0.001\end{array}$ \\
\hline
\end{tabular}

*:City and town were combined as one category in 1998 wave 
Table 5 DBP levels by seven waves

\begin{tabular}{|c|c|c|c|c|c|c|c|c|c|}
\hline Variable & Wave & 1998 & 2000 & 2002 & 2005 & 2008 & 2011 & 2014 & $p$ \\
\hline \multirow[t]{20}{*}{$\overline{\mathrm{DBP}}$} & \multicolumn{9}{|l|}{ Gender } \\
\hline & Male & $84.5 \pm 13.3$ & $81.9 \pm 12.8$ & $85.2 \pm 12.0$ & $82.5 \pm 11.7$ & $79.2 \pm 11.9$ & $79.2 \pm 11.8$ & $79.8 \pm 11.8$ & $<0.001$ \\
\hline & Female & $84.4 \pm 13.6$ & $81.7 \pm 13.0$ & $85.5 \pm 12.3$ & $81.8 \pm 12.1$ & $78.7 \pm 11.4$ & $79.7 \pm 12.5$ & $79.4 \pm 11.8$ & $<0.001$ \\
\hline & $\mathrm{p}$ & 0.854 & 0.419 & 0.216 & 0.007 & 0.031 & 0.126 & 0.285 & \\
\hline & \multicolumn{9}{|c|}{ Age-group } \\
\hline & $80-89$ yrs & $85.5 \pm 13.3$ & $82.1 \pm 13.6$ & $85.5 \pm 12.7$ & $83.2 \pm 12.2$ & $79.0 \pm 11.6$ & $80.2 \pm 11.8$ & $80.3 \pm 11.4$ & $<0.001$ \\
\hline & $90-99$ yrs & $84.7 \pm 13.4$ & $81.9 \pm 13.1$ & $85.5 \pm 11.9$ & $81.9 \pm 11.9$ & $79.0 \pm 12.0$ & $79.4 \pm 12.2$ & $79.2 \pm 12.0$ & $<0.001$ \\
\hline & $100-y r s$ & $82.6 \pm 13.7$ & $81.0 \pm 12.3$ & $85.0 \pm 11.8$ & $80.9 \pm 11.8$ & $78.6 \pm 11.2$ & $78.4 \pm 13.4$ & $78.6 \pm 12.3$ & 0.854 \\
\hline & $\mathrm{p}_{\text {for trend }}$ & $<0.001$ & 0.003 & 0.119 & $<0.001$ & 0.121 & $<0.001$ & $<0.001$ & \\
\hline & \multicolumn{9}{|c|}{ Category of residence } \\
\hline & City & $83.6 \pm 13.0$ & $81.5 \pm 14.0$ & $84.3 \pm 12.2$ & $81.7 \pm 11.7$ & $79.6 \pm 12.5$ & $77.4 \pm 12.3$ & $77.3 \pm 12.0$ & $<0.001$ \\
\hline & Town & & $81.7 \pm 13.0$ & $85.7 \pm 12.2$ & $81.8 \pm 12.4$ & $78.8 \pm 11.7$ & $80.0 \pm 11.9$ & $79.6 \pm 11.2$ & $<0.001$ \\
\hline & Rural & $84.9 \pm 13.7$ & $82.2 \pm 11.9$ & $85.8 \pm 12.1$ & $83.4 \pm 11.9$ & $78.9 \pm 11.2$ & $80.2 \pm 12.8$ & $80.2 \pm 12.0$ & $<0.001$ \\
\hline & $\mathrm{p}_{\text {for trend }}$ & $<0.001$ & 0.044 & $<0.001$ & $<0.001$ & $<0.001$ & $<0.001$ & $<0.001$ & \\
\hline & \multicolumn{9}{|c|}{ Region of China } \\
\hline & East & $85.7 \pm 12.8$ & $82.5 \pm 12.9$ & $85.1 \pm 11.4$ & $84.0 \pm 10.8$ & $79.5 \pm 10.9$ & $80.6 \pm 11.7$ & $81.0 \pm 11.6$ & $<0.001$ \\
\hline & Central & $84.1 \pm 14.2$ & $82.1 \pm 13.4$ & $84.6 \pm 12.0$ & $80.1 \pm 13.7$ & $79.4 \pm 12.9$ & $80.2 \pm 12.3$ & $80.2 \pm 12.4$ & $<0.001$ \\
\hline & West & $82.9 \pm 14.4$ & $79.5 \pm 12.7$ & $82.6 \pm 12.3$ & $79.1 \pm 11.0$ & $76.5 \pm 11.8$ & $78.6 \pm 12.2$ & $79.0 \pm 11.4$ & $<0.001$ \\
\hline & $p_{\text {for trend }}$ & $<0.001$ & $<0.001$ & $<0.001$ & $<0.001$ & $<0.001$ & $<0.001$ & $<0.001$ & \\
\hline & Total & $84.3 \pm 13.4$ & $81.8 \pm 13.0$ & $84.6 \pm 11.7$ & $82.0 \pm 11.9$ & $78.9 \pm 11.7$ & $79.4 \pm 12.0$ & $79.7 \pm 11.8$ & $<0.001$ \\
\hline \multirow[t]{20}{*}{ DBP among those without hypertension } & \multicolumn{9}{|l|}{ Gender } \\
\hline & Male & $80.3 \pm 10.7$ & $78.1 \pm 9.6$ & $80.6 \pm 8.2$ & $76.6 \pm 7.3$ & $75.3 \pm 8.7$ & $73.9 \pm 8.8$ & $74.5 \pm 8.0$ & $<0.001$ \\
\hline & Female & $80.0 \pm 11.0$ & $77.8 \pm 9.5$ & $80.3 \pm 8.1$ & $75.6 \pm 7.9$ & $75.3 \pm 8.6$ & $74.6 \pm 9.0$ & $73.7 \pm 8.8$ & $<0.001$ \\
\hline & $\mathrm{p}$ & 0.305 & 0.184 & 0.120 & $<0.001$ & 0.794 & 0.323 & 0.034 & \\
\hline & \multicolumn{9}{|c|}{ Age-group } \\
\hline & 80-89yrs & $81.3 \pm 10.6$ & $78.1 \pm 9.6$ & $80.6 \pm 8.1$ & $76.7 \pm 7.5$ & $75.8 \pm 8.5$ & $74.2 \pm 8.4$ & $74.9 \pm 7.9$ & $<0.001$ \\
\hline & $90-99$ yrs & $80.3 \pm 10.7$ & $78.1 \pm 9.4$ & $80.4 \pm 8.0$ & $76.1 \pm 7.6$ & $75.2 \pm 8.5$ & $73.9 \pm 8.9$ & $73.7 \pm 8.7$ & $<0.001$ \\
\hline & $100-y r s$ & $78.4 \pm 11.2$ & $77.2 \pm 9.7$ & $80.4 \pm 8.3$ & $75.1 \pm 7.9$ & $75.0 \pm 8.8$ & $72.8 \pm 9.5$ & $73.0 \pm 9.2$ & 0.712 \\
\hline & $p_{\text {for trend }}$ & $<0.001$ & 0.011 & 0.652 & $<0.001$ & 0.009 & 0.003 & $<0.001$ & \\
\hline & \multicolumn{9}{|c|}{ Category of residence } \\
\hline & City & $79.9 \pm 10.6$ & $77.7 \pm 10.0$ & $80.1 \pm 8.5$ & $75.5 \pm 7.3$ & $75.1 \pm 8.9$ & $72.4 \pm 9.2$ & $72.9 \pm 8.6$ & $<0.001$ \\
\hline & Town & & $78.0 \pm 9.6$ & $80.5 \pm 7.8$ & $76.0 \pm 7.5$ & $75.5 \pm 8.3$ & $73.8 \pm 8.3$ & $74.2 \pm 8.5$ & $<0.001$ \\
\hline & Rural & $80.2 \pm 11.0$ & $78.0 \pm 9.3$ & $80.7 \pm 8.1$ & $76.4 \pm 7.9$ & $75.8 \pm 8.6$ & $74.5 \pm 9.1$ & $74.4 \pm 8.4$ & $<0.001$ \\
\hline & $\mathrm{p}_{\text {for trend }}$ & 0.321 & 0.684 & 0.112 & 0.019 & 0.063 & $<0.001$ & 0.038 & \\
\hline & \multicolumn{9}{|c|}{ Region of China } \\
\hline & East & $81.0 \pm 10.5$ & $78.3 \pm 9.8$ & $80.9 \pm 7.9$ & $77.6 \pm 6.5$ & $76.2 \pm 8.0$ & $74.5 \pm 8.9$ & $74.9 \pm 8.6$ & $<0.001$ \\
\hline & Central & $80.2 \pm 11.2$ & $77.9 \pm 9.0$ & $80.7 \pm 8.6$ & $75.4 \pm 8.7$ & $75.2 \pm 9.4$ & $73.8 \pm 9.2$ & $74.4 \pm 8.6$ & $<0.001$ \\
\hline & West & $78.2 \pm 11.7$ & $76.5 \pm 9.5$ & $78.0 \pm 7.4$ & $73.3 \pm 7.9$ & $71.7 \pm 9.3$ & $73.4 \pm 8.4$ & $74.0 \pm 7.8$ & $<0.001$ \\
\hline & $p_{\text {for trend }}$ & $<0.001$ & $<0.001$ & $<0.001$ & $<0.001$ & $<0.001$ & 0.057 & 0.265 & \\
\hline & Total & $80.0 \pm 10.9$ & $77.9 \pm 9.6$ & $80.4 \pm 8.1$ & $76.0 \pm 7.7$ & $75.3 \pm 8.7$ & $73.7 \pm 8.9$ & $74.2 \pm 8.6$ & $<0.001$ \\
\hline
\end{tabular}


Table 6 MAP levels by seven waves

\begin{tabular}{|c|c|c|c|c|c|c|c|c|c|}
\hline Variable & Wave & 1998 & 2000 & 2002 & 2005 & 2008 & 2011 & 2014 & $p$ \\
\hline \multirow[t]{20}{*}{$\overline{M A P}$} & \multicolumn{9}{|l|}{ Gender } \\
\hline & Male & $\begin{array}{l}105.7 \pm \\
14.9\end{array}$ & $\begin{array}{l}100.4 \pm \\
13.4\end{array}$ & $\begin{array}{l}101.7 \pm \\
12.0\end{array}$ & $\begin{array}{l}98.8 \pm \\
11.8\end{array}$ & $\begin{array}{l}98.0 \pm \\
12.4\end{array}$ & $\begin{array}{l}97.9 \pm \\
13.0\end{array}$ & $99.5 \pm 13.0$ & $\begin{array}{l}< \\
0.001\end{array}$ \\
\hline & Female & $\begin{array}{l}105.9 \pm \\
15.5\end{array}$ & $\begin{array}{l}100.3 \pm \\
13.9\end{array}$ & $\begin{array}{l}101.7 \pm \\
12.4\end{array}$ & $\begin{array}{l}98.0 \pm \\
12.6\end{array}$ & $\begin{array}{l}97.8 \pm \\
12.0\end{array}$ & $\begin{array}{l}99.2 \pm \\
14.2\end{array}$ & $99.6 \pm 13.5$ & $\begin{array}{l}< \\
0.001\end{array}$ \\
\hline & $p$ & 0.405 & 0.695 & 0.835 & 0.001 & 0.551 & $<0.001$ & 0.784 & \\
\hline & \multicolumn{9}{|c|}{ Age-group } \\
\hline & $\begin{array}{l}80-89 \\
\text { yrs }\end{array}$ & $\begin{array}{l}107.3 \pm \\
15.0\end{array}$ & $\begin{array}{l}100.8 \pm \\
13.9\end{array}$ & $\begin{array}{l}102.2 \pm \\
12.7\end{array}$ & $\begin{array}{l}99.6 \pm \\
12.5\end{array}$ & $\begin{array}{l}98.7 \pm \\
12.5\end{array}$ & $\begin{array}{l}99.4 \pm \\
13.1\end{array}$ & $\begin{array}{l}100.3 \pm \\
12.7\end{array}$ & $\begin{array}{l}< \\
0.001\end{array}$ \\
\hline & $\begin{array}{l}90-99 \\
\text { yrs }\end{array}$ & $\begin{array}{l}106.0 \pm \\
15.1\end{array}$ & $\begin{array}{l}100.7 \pm \\
13.9\end{array}$ & $\begin{array}{l}101.8 \pm \\
11.9\end{array}$ & $\begin{array}{l}98.3 \pm \\
12.1\end{array}$ & $\begin{array}{l}97.8 \pm \\
12.5\end{array}$ & $\begin{array}{l}98.8 \pm \\
13.9\end{array}$ & $99.2 \pm 13.5$ & $\begin{array}{l}< \\
0.001\end{array}$ \\
\hline & $100-y r s$ & $\begin{array}{l}103.3 \pm \\
15.7\end{array}$ & $99.0 \pm 12.9$ & $\begin{array}{l}101.0 \pm \\
11.9\end{array}$ & $\begin{array}{l}96.5 \pm \\
12.0\end{array}$ & $\begin{array}{l}96.8 \pm \\
11.1\end{array}$ & $\begin{array}{l}97.2 \pm \\
14.6\end{array}$ & $98.3 \pm 14.3$ & 0.247 \\
\hline & $\mathrm{p}_{\text {for trend }}$ & $<0.001$ & $<0.001$ & $<0.001$ & $<0.001$ & $<0.001$ & $<0.001$ & $<0.001$ & \\
\hline & \multicolumn{9}{|c|}{ Category of residence } \\
\hline & City & $\begin{array}{l}105.2 \pm \\
14.7\end{array}$ & $\begin{array}{l}100.2 \pm \\
14.5\end{array}$ & $\begin{array}{l}101.2 \pm \\
12.3\end{array}$ & $\begin{array}{l}98.0 \pm \\
12.3\end{array}$ & $\begin{array}{l}97.5 \pm \\
11.9\end{array}$ & $\begin{array}{l}96.0 \pm \\
13.3\end{array}$ & $96.2 \pm 12.8$ & $\begin{array}{l}< \\
0.001\end{array}$ \\
\hline & Town & & $\begin{array}{l}100.3 \pm \\
13.6\end{array}$ & $\begin{array}{l}101.8 \pm \\
12.4\end{array}$ & $\begin{array}{l}98.3 \pm \\
12.7\end{array}$ & $\begin{array}{l}97.8 \pm \\
12.1\end{array}$ & $\begin{array}{l}99.0 \pm \\
13.4\end{array}$ & $99.4 \pm 12.4$ & $\begin{array}{l}< \\
0.001\end{array}$ \\
\hline & Rural & $\begin{array}{l}106.1 \pm \\
15.6\end{array}$ & $\begin{array}{l}100.6 \pm \\
13.1\end{array}$ & $\begin{array}{l}102.0 \pm \\
12.1\end{array}$ & $\begin{array}{l}99.1 \pm \\
12.3\end{array}$ & $\begin{array}{l}98.0 \pm \\
12.3\end{array}$ & $\begin{array}{l}99.4 \pm \\
14.0\end{array}$ & $\begin{array}{l}100.5 \pm \\
13.7\end{array}$ & $\begin{array}{l}< \\
0.001\end{array}$ \\
\hline & $\mathrm{P}_{\text {for trend }}$ & 0.005 & 0.451 & 0.034 & 0.008 & 0.214 & $<0.001$ & $<0.001$ & \\
\hline & \multicolumn{9}{|c|}{ Region of China } \\
\hline & East & $\begin{array}{l}106.5 \pm \\
14.7\end{array}$ & $\begin{array}{l}101.0 \pm \\
13.4\end{array}$ & $\begin{array}{l}101.6 \pm \\
11.7\end{array}$ & $\begin{array}{l}99.5 \pm \\
11.5\end{array}$ & $\begin{array}{l}98.1 \pm \\
11.6\end{array}$ & $\begin{array}{l}98.8 \pm \\
13.3\end{array}$ & $\begin{array}{l}100.6 \pm \\
13.1\end{array}$ & $\begin{array}{l}< \\
0.001\end{array}$ \\
\hline & Central & $\begin{array}{l}105.7 \pm \\
15.8\end{array}$ & $99.9 \pm 14.5$ & $\begin{array}{l}101.1 \pm \\
12.3\end{array}$ & $\begin{array}{l}97.0 \pm \\
13.8\end{array}$ & $\begin{array}{l}98.0 \pm \\
13.2\end{array}$ & $\begin{array}{l}98.5 \pm \\
14.0\end{array}$ & $99.4 \pm 13.9$ & $\begin{array}{l}< \\
0.001\end{array}$ \\
\hline & West & $\begin{array}{l}104.1 \pm \\
16.1\end{array}$ & $97.3 \pm 13.2$ & $99.1 \pm 12.0$ & $\begin{array}{l}96.0 \pm \\
11.2\end{array}$ & $\begin{array}{l}97.6 \pm \\
12.7\end{array}$ & $\begin{array}{l}98.2 \pm \\
13.6\end{array}$ & $99.0 \pm 12.2$ & $\begin{array}{l}< \\
0.001\end{array}$ \\
\hline & $p_{\text {for trend }}$ & $<0.001$ & $<0.001$ & $<0.001$ & $<0.001$ & $<0.001$ & 0.459 & 0.020 & $\begin{array}{l}< \\
0.001\end{array}$ \\
\hline & Total & $\begin{array}{l}105.6 \pm \\
15.2\end{array}$ & $\begin{array}{l}100.2 \pm \\
13.7\end{array}$ & $\begin{array}{l}101.1 \pm \\
12.0\end{array}$ & $\begin{array}{l}98.2 \pm \\
12.2\end{array}$ & $\begin{array}{l}98.0 \pm \\
12.3\end{array}$ & $\begin{array}{l}98.6 \pm \\
13.6\end{array}$ & $99.7 \pm 13.3$ & $\begin{array}{l}< \\
0.001\end{array}$ \\
\hline \multirow{12}{*}{$\begin{array}{l}\text { MAP among those without } \\
\text { hypertension }\end{array}$} & \multicolumn{9}{|l|}{ Gender } \\
\hline & Male & $98.8 \pm 12.2$ & $94.1 \pm 10.5$ & $95.9 \pm 8.7$ & $91.2 \pm 7.1$ & $91.1 \pm 8.0$ & $89.8 \pm 8.6$ & $90.7 \pm 7.8$ & $\begin{array}{l}< \\
0.001\end{array}$ \\
\hline & Female & $98.7 \pm 13.0$ & $93.7 \pm 10.6$ & $95.3 \pm 8.6$ & $90.1 \pm 7.7$ & $91.1 \pm 7.8$ & $89.3 \pm 8.9$ & $89.8 \pm 8.5$ & $\begin{array}{l}< \\
0.001\end{array}$ \\
\hline & $p$ & 0.732 & 0.107 & 0.006 & $<0.001$ & 0.990 & 0.106 & 0.007 & \\
\hline & \multicolumn{9}{|c|}{ Age-group } \\
\hline & $\begin{array}{l}80-89 \\
\text { yrs }\end{array}$ & $\begin{array}{l}100.4 \pm \\
12.3\end{array}$ & $94.3 \pm 10.8$ & $95.7 \pm 8.7$ & $91.3 \pm 7.3$ & $91.0 \pm 7.7$ & $90.3 \pm 8.2$ & $91.2 \pm 7.5$ & $\begin{array}{l}< \\
0.001\end{array}$ \\
\hline & $\begin{array}{l}90-99 \\
\text { yrs }\end{array}$ & $98.8 \pm 12.4$ & $94.1 \pm 10.5$ & $95.7 \pm 8.6$ & $90.8 \pm 7.2$ & $90.8 \pm 7.9$ & $89.6 \pm 8.8$ & $90.0 \pm 8.3$ & $\begin{array}{l}< \\
0.001\end{array}$ \\
\hline & $100-y r s$ & $96.5 \pm 13.2$ & $92.8 \pm 10.2$ & $95.2 \pm 8.8$ & $89.3 \pm 7.9$ & $91.4 \pm 7.9$ & $88.2 \pm 9.4$ & $88.5 \pm 9.3$ & $\begin{array}{l}< \\
0.001\end{array}$ \\
\hline & $p_{\text {for trend }}$ & $<0.001$ & $<0.001$ & 0.112 & $<0.001$ & 0.099 & $<0.001$ & $<0.001$ & \\
\hline & \multicolumn{9}{|c|}{ Category of residence } \\
\hline & City & $98.7 \pm 12.4$ & $94.0 \pm 10.9$ & $95.5 \pm 9.3$ & $90.0 \pm 7.3$ & $90.9 \pm 7.8$ & $88.4 \pm 8.8$ & $88.9 \pm 8.5$ & $\begin{array}{l}< \\
0.001\end{array}$ \\
\hline & Town & & $94.0 \pm 10.3$ & $95.5 \pm 8.4$ & $90.6 \pm 7.6$ & $91.2 \pm 7.5$ & $89.5 \pm 8.3$ & $90.3 \pm 8.4$ & $<$ \\
\hline
\end{tabular}


Table 6 MAP levels by seven waves (Continued)

\begin{tabular}{|c|c|c|c|c|c|c|c|c|c|}
\hline Variable & Wave & 1998 & 2000 & 2002 & 2005 & 2008 & 2011 & 2014 & $p$ \\
\hline & & & & & & & & & 0.001 \\
\hline & Rural & $98.8 \pm 12.9$ & $97.8 \pm 10.5$ & $95.7 \pm 8.5$ & $90.7 \pm 7.5$ & $91.3 \pm 8.0$ & $90.3 \pm 8.9$ & $90.7 \pm 8.1$ & $\begin{array}{l}< \\
0.001\end{array}$ \\
\hline & $p_{\text {for trend }}$ & 0.821 & 0.795 & 0.742 & 0.011 & 0.340 & $<0.001$ & 0.005 & \\
\hline & \multicolumn{9}{|c|}{ Region of China } \\
\hline & East & $99.5 \pm 13.1$ & $94.6 \pm 10.8$ & $96.0 \pm 8.6$ & $91.8 \pm 6.6$ & $91.9 \pm 7.3$ & $90.3 \pm 8.3$ & $91.1 \pm 7.7$ & $\begin{array}{l}< \\
0.001\end{array}$ \\
\hline & Central & $98.9 \pm 12.3$ & $93.2 \pm 10.2$ & $95.8 \pm 9.2$ & $90.4 \pm 8.0$ & $90.4 \pm 8.5$ & $89.7 \pm 8.5$ & $90.2 \pm 8.3$ & $\begin{array}{l}< \\
0.001\end{array}$ \\
\hline & West & $96.7 \pm 13.2$ & $92.2 \pm 9.6$ & $93.2 \pm 8.0$ & $88.3 \pm 8.1$ & $88.6 \pm 8.3$ & $88.8 \pm 9.2$ & $90.2 \pm 8.4$ & $\begin{array}{l}< \\
0.001\end{array}$ \\
\hline & $\mathrm{p}_{\text {for trend }}$ & $<0.001$ & $<0.001$ & $<0.001$ & $<0.001$ & $<0.001$ & $<0.001$ & 0.316 & \\
\hline & Total & $98.7 \pm 12.7$ & $93.8 \pm 10.5$ & $95.5 \pm 8.7$ & $90.6 \pm 7.5$ & $91.0 \pm 7.9$ & $89.6 \pm 8.7$ & $90.3 \pm 8.3$ & $\begin{array}{l}< \\
0.001\end{array}$ \\
\hline
\end{tabular}


Table 7 PP levels by seven waves

\begin{tabular}{|c|c|c|c|c|c|c|c|c|c|}
\hline Variable & Wave & 1998 & 2000 & 2002 & 2005 & 2008 & 2011 & 2014 & $p$ \\
\hline \multirow[t]{20}{*}{$\overline{\mathrm{PP}}$} & \multicolumn{9}{|l|}{ Gender } \\
\hline & Male & $63.4 \pm 19.1$ & $55.6 \pm 18.8$ & $49.6 \pm 15.0$ & $48.9 \pm 16.5$ & $56.3 \pm 19.9$ & $56.0 \pm 16.8$ & $59.1 \pm 17.6$ & $<0.001$ \\
\hline & Female & $64.4 \pm 20.3$ & $55.9 \pm 19.5$ & $48.8 \pm 14.8$ & $48.5 \pm 16.5$ & $57.4 \pm 20.4$ & $58.4 \pm 18.8$ & $60.6 \pm 19.4$ & $<0.001$ \\
\hline & $p$ & 0.021 & 0.427 & 0.012 & 0.195 & 0.008 & $<0.001$ & 0.009 & \\
\hline & \multicolumn{9}{|l|}{ Age-group } \\
\hline & $80-89$ yrs & $65.4 \pm 19.9$ & $56.6 \pm 19.6$ & $49.9 \pm 15.3$ & $49.2 \pm 16.5$ & $59.0 \pm 20.3$ & $58.0 \pm 17.0$ & $60.2 \pm 17.8$ & $<0.001$ \\
\hline & 90-99yrs & $63.9 \pm 19.6$ & $55.8 \pm 19.4$ & $49.0 \pm 15.0$ & $49.2 \pm 17.0$ & $56.5 \pm 20.3$ & $57.7 \pm 18.6$ & $60.1 \pm 19.3$ & $<0.001$ \\
\hline & $100-y r s$ & $62.1 \pm 19.9$ & $54.0 \pm 18.1$ & $48.1 \pm 14.5$ & $47.1 \pm 15.7$ & $55.0 \pm 20.0$ & $56.0 \pm 19.0$ & $59.0 \pm 19.8$ & 0.247 \\
\hline & $p_{\text {for trend }}$ & $<0.001$ & $<0.001$ & $<0.001$ & $<0.001$ & $<0.001$ & 0.004 & 0.244 & \\
\hline & \multicolumn{9}{|c|}{ Category of residence } \\
\hline & City & $63.6 \pm 19.9$ & $55.1 \pm 19.0$ & $48.5 \pm 14.6$ & $46.9 \pm 15.8$ & $54.0 \pm 18.9$ & $55.8 \pm 16.6$ & $56.3 \pm 17.1$ & $<0.001$ \\
\hline & Town & & $55.5 \pm 18.7$ & $48.8 \pm 14.2$ & $49.1 \pm 15.4$ & $55.8 \pm 19.5$ & $56.5 \pm 17.0$ & $59.8 \pm 17.9$ & $<0.001$ \\
\hline & Rural & $64.6 \pm 19.8$ & $56.5 \pm 19.7$ & $50.8 \pm 16.1$ & $49.1 \pm 17.2$ & $58.3 \pm 20.7$ & $58.5 \pm 19.0$ & $61.1 \pm 19.4$ & $<0.001$ \\
\hline & $p_{\text {for trend }}$ & 0.024 & 0.008 & $<0.001$ & $<0.001$ & $<0.001$ & $<0.001$ & $<0.001$ & \\
\hline & \multicolumn{9}{|c|}{ Region of China } \\
\hline & East & $64.9 \pm 19.5$ & $56.9 \pm 19.0$ & $49.6 \pm 14.4$ & $53.7 \pm 20.3$ & $63.2 \pm 21.6$ & $60.6 \pm 19.0$ & $61.1 \pm 19.5$ & $<0.001$ \\
\hline & Central & $63.7 \pm 20.0$ & $53.5 \pm 18.5$ & $49.5 \pm 16.1$ & $47.8 \pm 14.2$ & $56.0 \pm 20.9$ & $53.8 \pm 17.0$ & $58.8 \pm 18.0$ & $<0.001$ \\
\hline & West & $62.4 \pm 20.4$ & $52.3 \pm 18.6$ & $49.4 \pm 15.2$ & $46.7 \pm 14.7$ & $55.9 \pm 19.5$ & $53.7 \pm 14.5$ & $56.5 \pm 16.3$ & $<0.001$ \\
\hline & $\mathrm{p}_{\text {for trend }}$ & $<0.001$ & 0.908 & $<0.001$ & $<0.001$ & $<0.001$ & $<0.001$ & $<0.001$ & $<0.001$ \\
\hline & Total & $64.1 \pm 19.8$ & $55.2 \pm 18.9$ & $49.5 \pm 15.0$ & $48.8 \pm 16.6$ & $57.1 \pm 20.5$ & $57.7 \pm 18.1$ & $59.9 \pm 18.8$ & $<0.001$ \\
\hline \multirow[t]{20}{*}{ PP among those without hypertension } & \multicolumn{9}{|l|}{ Gender } \\
\hline & Male & $55.4 \pm 15.7$ & $48.2 \pm 14.1$ & $45.7 \pm 12.6$ & $43.8 \pm 10.3$ & $47.4 \pm 11.3$ & $47.7 \pm 10.2$ & $48.8 \pm 9.7$ & $<0.001$ \\
\hline & Female & $56.0 \pm 16.7$ & $47.8 \pm 14.8$ & $44.9 \pm 11.9$ & $43.4 \pm 10.5$ & $47.2 \pm 11.7$ & $47.1 \pm 10.8$ & $48.2 \pm 11.0$ & $<0.001$ \\
\hline & $\mathrm{p}$ & 0.209 & 0.377 & 0.006 & 0.189 & 0.543 & 0.127 & 0.247 & \\
\hline & \multicolumn{9}{|c|}{ Age-group } \\
\hline & $80-89$ yrs & $57.2 \pm 16.2$ & $48.6 \pm 15.1$ & $45.9 \pm 12.6$ & $44.2 \pm 10.4$ & $48.2 \pm 11.7$ & $48.1 \pm 10.3$ & $49.0 \pm 9.8$ & $<0.001$ \\
\hline & 90-99yrs & $55.5 \pm 16.1$ & $48.1 \pm 14.7$ & $45.3 \pm 12.3$ & $43.8 \pm 10.3$ & $46.9 \pm 11.4$ & $47.3 \pm 10.4$ & $48.8 \pm 10.9$ & $<0.001$ \\
\hline & $100-y r s$ & $54.4 \pm 16.1$ & $46.6 \pm 13.0$ & $44.3 \pm 11.5$ & $42.6 \pm 10.4$ & $46.7 \pm 11.5$ & $46.3 \pm 11.0$ & $46.6 \pm 11.0$ & 0.611 \\
\hline & $p_{\text {for trend }}$ & $<0.001$ & $<0.001$ & $<0.001$ & $<0.001$ & $<0.001$ & $<0.001$ & $<0.001$ & \\
\hline & \multicolumn{9}{|c|}{ Category of residence } \\
\hline & City & $55.5 \pm 16.3$ & $47.9 \pm 14.3$ & $44.9 \pm 12.0$ & $42.6 \pm 9.6$ & $46.6 \pm 11.4$ & $47.1 \pm 11.0$ & $47.8 \pm 10.1$ & $<0.001$ \\
\hline & Town & & $48.0 \pm 14.1$ & $45.0 \pm 11.8$ & $43.3 \pm 10.5$ & $47.2 \pm 11.6$ & $47.4 \pm 10.0$ & $48.3 \pm 10.5$ & $<0.001$ \\
\hline & Rural & $56.3 \pm 16.4$ & $48.0 \pm 15.1$ & $46.3 \pm 12.8$ & $44.0 \pm 10.6$ & $47.5 \pm 11.6$ & $48.1 \pm 10.7$ & $49.0 \pm 10.6$ & $<0.001$ \\
\hline & $\mathrm{p}_{\text {for trend }}$ & 0.012 & 0.960 & 0.001 & $<0.001$ & 0.059 & 0.150 & 0.210 & \\
\hline & \multicolumn{9}{|c|}{ Region of China } \\
\hline & East & $56.3 \pm 15.9$ & $49.1 \pm 14.6$ & $45.7 \pm 11.4$ & $42.4 \pm 10.2$ & $47.3 \pm 10.7$ & $49.0 \pm 10.6$ & $48.7 \pm 10.4$ & $<0.001$ \\
\hline & Central & $55.5 \pm 17.3$ & $45.7 \pm 14.2$ & $45.3 \pm 14.3$ & $45.2 \pm 10.0$ & $45.5 \pm 12.3$ & $44.9 \pm 10.6$ & $48.4 \pm 10.2$ & $<0.001$ \\
\hline & West & $55.3 \pm 16.1$ & $47.3 \pm 13.5$ & $45.2 \pm 11.3$ & $44.8 \pm 11.0$ & $50.7 \pm 13.1$ & $47.4 \pm 9.9$ & $47.4 \pm 10.6$ & $<0.001$ \\
\hline & $\mathrm{p}$ for trend & 0.190 & $<0.001$ & 0.514 & $<0.001$ & $<0.001$ & $<0.001$ & 0.078 & \\
\hline & Total & $55.9 \pm 16.3$ & $47.8 \pm 14.4$ & $45.3 \pm 12.2$ & $43.6 \pm 10.4$ & $47.2 \pm 11.7$ & $47.5 \pm 10.6$ & $48.3 \pm 10.4$ & $<0.001$ \\
\hline
\end{tabular}


Table 8 The prevalence (\%) of ISH by seven waves

\begin{tabular}{|c|c|c|c|c|c|c|c|c|}
\hline$\overline{\text { Wave }}$ & 1998 & 2000 & 2002 & 2005 & 2008 & 2011 & 2014 & $p$ \\
\hline \multicolumn{9}{|l|}{ Gender } \\
\hline Male & $30.6(29.0-32.1)$ & $24.8(23.6-26.1)$ & $15.3(14.2-16.3)$ & $15.0(13.9-16.1)$ & $26.5(25.2-27.8)$ & $50.1(48.1-52.1)$ & $29.0(26.9-31.0)$ & $<0.001$ \\
\hline Female & $30.5(29.2-31.7)$ & $25.2(24.1-26.3)$ & 13.7(12.9-14.6) & 13.8(13.0-14.7) & $28.2(27.1-29.3)$ & 23.5(21.9-25.2) & $31.9(30.1-33.6)$ & $<0.001$ \\
\hline$p$ & 0.930 & 0.669 & 0.025 & 0.100 & 0.054 & $<0.001$ & 0.036 & \\
\hline \multicolumn{9}{|l|}{ Age-group } \\
\hline $80-89$ yrs & $31.3(29.7-32.8)$ & $25.1(23.9-26.3)$ & $15.1(14.0-16.1)$ & $15.6(14.4-16.7)$ & $30.4(29.0-31.8)$ & $26.2(24.5-27.9)$ & $30.8(28.832 .7)$ & $<0.001$ \\
\hline 90-99 yrs & $30.1(28.5-31.8)$ & $25.6(24.2-27.0)$ & $14.2(13.1-15.3)$ & 14.8(13.7-15.9) & $24.0(26.0-28.7)$ & 27.1(25.4-28.9) & $30.5(28.2-32.7)$ & $<0.001$ \\
\hline $100-y r s$ & $30.0(28.1-31.8)$ & $24.1(22.3-25.8)$ & $13.5(12.3-14.7)$ & $11.7(10.5-12.9)$ & $23.4(22.4-25.5)$ & $23.3(21.0-25.5)$ & $30.7(27.6-33.9)$ & $<0.001$ \\
\hline$p_{\text {for trend }}$ & 0.269 & 0.491 & 0.043 & $<0.001$ & $<0.001$ & 0.098 & 0.941 & \\
\hline \multicolumn{9}{|c|}{ Category of residence } \\
\hline City & $29.1(27.9-30.3)$ & $22.8(21.4-24.3)$ & 13.5(12.7-14.4) & $11.6(10.3-13.0)$ & $23.0(21.3-24.7)$ & $22.4(19.9-24.9)$ & $26.9(23.4-30.4)$ & $<0.001$ \\
\hline Town & & $25.0(23.5-26.4)$ & $14.2(12.9-15.6)$ & $14.6(13.7-15.5)$ & $26.2(24.4-28.1)$ & $24.2(22.3-26.1)$ & $31.4(29.6-33.2)$ & $<0.001$ \\
\hline Rural & $33.0(31.4-34.6)$ & $26.9(25.6-28.3)$ & $16.2(14.8-17.6)$ & $15.8(14.4-17.2)$ & 29.5(28.4-30.6) & $28.0(26.5-29.5)$ & $30.9(28.5-33.3)$ & $<0.001$ \\
\hline$p$ for trend & $<0.001$ & $<0.001$ & 0.005 & $<0.001$ & $<0.001$ & $<0.001$ & 0.084 & \\
\hline \multicolumn{9}{|l|}{ Region of China } \\
\hline East & $32.4(31.3-33.8)$ & $27.1(25.9-28.2)$ & $16.1(14.7-17.5)$ & $21.1(19.5-22.6)$ & $31.0(29.9-32.1)$ & $31.7(30.1-33.2)$ & $32.4(30.5-34.2)$ & $<0.001$ \\
\hline Central & $30.3(27.9-32.8)$ & 19.4(17.9-20.9) & $14.9(14.0-15.8)$ & $14.1(12.4-15.7)$ & $27.9(26.3-29.4)$ & $20.7(18.8-22.6)$ & $28.7(26.2-31.1)$ & $<0.001$ \\
\hline West & $27.5(25.5-29.6)$ & $19.2(17.2-21.2)$ & $14.1(12.4-15.8)$ & $11.4(10.4-12.2)$ & $20.3(18.0-22.6)$ & $17.0(14.5-19.6)$ & $26.3(22.4-30.2)$ & $<0.001$ \\
\hline $\mathrm{P}_{\text {for trend }}$ & $<0.001$ & $<0.001$ & 0.153 & $<0.001$ & $<0.001$ & $<0.001$ & 0.002 & \\
\hline Total & $30.5(29.6-31.5)$ & $25.1(24.2-25.9)$ & $14.3(13.7-15.0)$ & $14.3(13.6-14.9)$ & $27.5(26.7-28.4)$ & $25.9(24.8-27.0)$ & $30.7(29.3-32.0)$ & $<0.001$ \\
\hline Weighted Total $^{+}$ & $30.5(29.5-31.4)$ & $26.6(25.8-27.4)$ & $14.0(12.7-15.4)$ & $14.8(13.4-16.2)$ & $30.2(28.4-32.0)$ & $23.7(21.9-25.6)$ & $30.1(27.9-32.2)$ & $<0.001$ \\
\hline Weighted Total $^{\dagger}$ & $31.2(30.2-32.1)$ & $25.2(24.4-26.0)$ & $14.9(14.2-15.6)$ & $15.4(14.7-16.1)$ & $30.1(29.3-31.0)$ & $26.5(25.4-27.5)$ & $30.9(29.6-32.2)$ & $<0.001$ \\
\hline
\end{tabular}

*:City and town were combined as one category in 1998 wave

${ }^{+}:$Weight was calculated based on age-sex-residence-specific distribution from the CLHLS study

${ }^{\ddagger}$ : Weight was calculated based on the sixth national census data

Table 9 BP levels by seven waves among those who were first included in each survey wave

\begin{tabular}{|c|c|c|c|c|c|c|c|c|}
\hline Variable & 1998 & 2000 & 2002 & 2005 & 2008 & 2011 & 2014 & $p$ \\
\hline \multicolumn{9}{|l|}{ mean $\pm S D$} \\
\hline SBP & $148.5 \pm 24.5$ & $137.6 \pm 21.8$ & $133.3 \pm 16.3$ & $130.6 \pm 18.7$ & $136.3 \pm 21.0$ & $139.6 \pm 22.6$ & $142.2 \pm 22.2$ & $<0.001$ \\
\hline DBP & $84.4 \pm 13.5$ & $81.8 \pm 12.9$ & $85.4 \pm 11.7$ & $82.1 \pm 12.1$ & $79.0 \pm 11.3$ & $79.7 \pm 11.8$ & $79.6 \pm 12.2$ & $<0.001$ \\
\hline MAP & $64.0 \pm 19.9$ & $55.8 \pm 19.2$ & $47.9 \pm 13.9$ & $48.6 \pm 16.4$ & $57.4 \pm 20.2$ & $59.9 \pm 19.0$ & $62.6 \pm 18.7$ & $<0.001$ \\
\hline PP & $105.8 \pm 15.3$ & $100.4 \pm 13.7$ & $101.4 \pm 11.6$ & $98.2 \pm 12.3$ & $98.1 \pm 11.9$ & $99.7 \pm 13.5$ & $100.5 \pm 13.6$ & $<0.001$ \\
\hline \multicolumn{9}{|l|}{$\%$} \\
\hline Prevalence & $43.1(42.0-44.1)$ & $43.6(42.7-44.6)$ & $41.2(39.4-42.9)$ & $47.1(46.1-48.1)$ & $46.4(45.3-47.6)$ & $58.8(56.5-61.0)$ & 62.3(59.7-64.9) & $<0.001$ \\
\hline
\end{tabular}




\section{Abbreviations}

BP: Blood pressure; Cl: Confidence interval; CLHLS: Chinese Longitudinal Healthy Longevity Survey; DBP: Diastolic blood pressure; MAP: Mean arterial pressure; NHANES: National Health and Nutrition Examination Survey; OR: Odds ratio; PP: Pulse pressure; SBP: Systolic blood pressure

\section{Acknowledgements}

We thank the staff from Center for Healthy Ageing and Development Studies for the data from the longitudinal health and Longevity Survey (CLSHL).

\section{Authors' contributions}

All of the authors have made great contributions to research design, field survey, data collection, data analysis, and drafting of the manuscript. JJD and GNZ were involved with data extraction, data analysis and interpretation and co-drafting of the manuscript. YHY was involved with data analysis and interpretation of the manuscript. ML was involved with data interpretation and co-drafting of the manuscript. YH was involved with data analysis and interpretation, and co-drafting of the manuscript. All authors have approved the manuscript submitted.

\section{Funding}

This work was supported by Beijing Nova Program (Z181100006218085), Opening Foundation of State Key Laboratory of Kidney Diseases (KF-01-115), Opening Foundation of National Clinical Research Center of Geriatrics (NCRCG-PLAGH-2017017), National Natural Science Foundation of China (81773502, 81703285), Beijing Natural Science Foundation (7174350), Special Foundation for the Cultivation of Excellent Youth Science in PLA General Hospital (2017-YQPY-003), Military Fund (15BJZ41, 17BJZ51), Medical Big Data Fund of Chinese PLA General Hospital (2018MBD-029).

The study sponsors played no role in study design, participant recruitment, data analysis, or paper preparation.

\section{Availability of data and materials}

All data used in this study was stored at http://opendata.pku.edu.cn and available upon request.

\section{Ethics approval and consent to participate}

The use of CLHLS data was approved by the Biomedical Ethics Committee of Peking University.

\section{Consent for publication}

Not applicable.

\section{Competing interests}

The authors have declared that no competing interests exist.

\section{Author details}

${ }^{1}$ Medical Information Center, Chinese PLA General Hospital, Beijing, China ${ }^{2}$ Oncology Department of the Second Medical Center, Chinese PLA general hospital, Beijing, China. ${ }^{3}$ Medical Service, National Defense Mobilization Department, China Military Commission, Beijing, China. ${ }^{4}$ Beijing Key Laboratory of Aging and Geriatrics, National Clinical Research Center for Geriatrics Diseases, Institute of Geriatrics, State Key Laboratory of Kidney Diseases, Second Medical Center of Chinese PLA General Hospital, Beijing, China.

Received: 22 April 2019 Accepted: 28 August 2019

Published online: 09 September 2019

\section{References}

1. Franklin SS, Lopez VA, Wong ND, et al. Single versus combined blood pressure components and risk for cardiovascular disease: the Framingham heart study. Circulation. 2009;119:243-50. https://doi.org/10.1161/ CIRCULATIONAHA.108.797936.

2. Kobori $H$, Nangaku $M, N a v a r ~ L G$, et al. The intrarenal renin-angiotensin system: from physiology to the pathobiology of hypertension and kidney disease. Pharmacol Rev. 2007:59:251-87. https://doi.org/10.1124/pr.59.3.3.

3. Fan JH, Wang JB, Wang SM, et al. Longitudinal change in blood pressure is associated with cardiovascular disease mortality in a Chinese cohort. Heart. 2018;104:1764-71. https://doi.org/10.1136/heartjnl-2017-312850.
4. Na RK, Kim HC. Prevalence and trends of isolated systolic hypertension among Korean adults: the Korea National Health and nutrition examination survey, 1998-2012. Korean Circulation Journal. 2015;45:492-9. https://doi. org/10.4070/kcj.2015.45.6.492

5. Staessen JA, Gasowski J, Wang JG, et al. Risks of untreated and treated isolated systolic hypertension in the elderly: meta-analysis of outcome trials. Lancet. 2000;355:865-72.

6. Lu J, Lu Y, Wang $X$, et al. Prevalence, awareness, treatment, and control of hypertension in China: data from 1.7 million adults in a population-based screening study (China PEACE million persons project). Lancet. 2017;390: 2549-58. https://doi.org/10.1016/S0140-6736(17)32478-9.

7. Wang J, Zhang L, Wang F, et al. Prevalence, awareness, treatment, and control of hypertension in China: results from a National Survey. Am J Hypertens. 2014;27:1355-61. https://doi.org/10.1093/ajh/hpu053.

8. Zeng Y. Introduction to the Chinese Longitudinal Healthy Longevity Survey (CLHLS): Springer Netherlands; 2008. doi: https://doi.org/10.1007/978-1-402 0-6752-5_2.

9. Gu D, Reynolds K, Wu X, et al. Prevalence, awareness, treatment, and control of hypertension in China. Hypertension. 2002;40:920-7.

10. Bromfield SG, Bowling CB, Tanner RM, et al. Trends in hypertension prevalence, awareness, treatment, and control among US adults 80 years and older, 1988-2010. J Clin Hypertens. 2014;16:270-6. https://doi.org/1 $0.1111 /$ jch.12281.

11. Wang $Z$, Wu Y, Zhao $L$, et al. Trends in prevalence, awareness, treatment and control of hypertension in the middle-aged population of China, 19921998. Hypertens Res. 2004;27:703-9. https://doi.org/10.1291/hypres.27.703.

12. Kim NR, Kim HC. Prevalence and trends of isolated systolic hypertension among Korean adults: the Korea National Health and nutrition examination survey, 1998-2012. Korean Circ J. 2015;45:492-9. https://doi.org/10.4070/kcj.2 015.45.6.492.

13. Liu F, Ma YT, Yang YN, et al. The prevalence of isolated systolic hypertension in adult populations from the Han, Uygur and Kazakh ethnic groups in Xinjiang, China. Blood Press. 2014;23:154-9. https://doi.org/10.31 09/08037051.2013.838827.

14. Liu X, Rodriguez CJ, Wang K. Prevalence and trends of isolated systolic hypertension among untreated adults in the United States. J Am Soc Hypertens. 2015;9:197-205. https://doi.org/10.1016/j.jash.2015.01.002.

15. Kokubo Y, Kamide K. High-normal blood pressure and the risk of cardiovascular disease. Circ J. 2009;73:1381-5. https://doi.org/10.1253/circj.cj09-0336.

16. Roberts CK, Vaziri ND, Barnard RJ. Protective effects of estrogen on genderspecific development of diet-induced hypertension. J Appl Physiol. 2001;91: 2005. https://doi.org/10.1016/S0167-8760(00)00145-8.

17. Salwe KJ, Kalyansundaram D, Bahurupi Y. A study on polypharmacy and potential drug-drug interactions among elderly patients admitted in Department of Medicine of a tertiary care hospital in Puducherry. J Clin Diagn Res. 2016;10:FC06-10. https://doi.org/10.7860/JCDR/2016/16284.7273.

18. Komiya $H$, Umegaki $H$, Asai $A$, et al. Factors associated with polypharmacy in elderly home-care patients. Geriatr Gerontol Int. 2018;18:33-41. https://doi. org/10.1111/ggi.13132.

19. Scondotto G, Pojero F, Pollina Addario S, et al. The impact of polypharmacy and drug interactions among the elderly population in Western Sicily, Italy. Aging Clin Exp Res. 2018;30:81-7. https://doi.org/10.1007/s40520-0170755-2.

20. Tang $Y$, Jiang $M, L i ~ D R$, et al. Association between awareness of harmful effects of smoking and smoking cessation advice provided by hospital chest physicians in Guangzhou, China: a multi-institutional cross-sectional survey. Respirology. 2013;18:790-6. https://doi.org/10.1111/resp.12091.

21. Jordan J, Yumuk V, Schlaich $M$, et al. Joint statement of the European Association for the Study of obesity and the European Society of Hypertension: obesity and difficult to treat arterial hypertension. J Hypertens. 2012;34:1047-55. https://doi.org/10.1097/HJH.0000000000001013.

\section{Publisher's Note}

Springer Nature remains neutral with regard to jurisdictional claims in published maps and institutional affiliations. 